\title{
Spectral characterization of laser- accelerated protons with CR-39 nuclear track detector
}

Cite as: Rev. Sci. Instrum. 89, 023302 (2018); https://doi.org/10.1063/1.5009587

Submitted: 17 October 2017 . Accepted: 22 January 2018 . Published Online: 07 February 2018

M. Seimetz (D), P. Bellido, P. García, P. Mur, A. Iborra, A. Soriano, T. Hülber (D), J. García López, M. C. Jiménez-Ramos, R. Lera, A. Ruiz-de la Cruz, I. Sánchez (D), R. Zaffino (D), L. Roso, and J. M. Benlloch

\section{ARTICLES YOU MAY BE INTERESTED IN}

CR-39 track detector calibration for $\mathrm{H}, \mathrm{He}$, and $\mathrm{C}$ ions from 0.1-0.5 MeV up to $5 \mathrm{MeV}$ for laser-induced nuclear fusion product identification

Review of Scientific Instruments 86, 083307 (2015); https://doi.org/10.1063/1.4927684

The response of CR-39 nuclear track detector to 1-9 MeV protons

Review of Scientific Instruments 82, 103303 (2011); https://doi.org/10.1063/1.3653549

Demonstration of laser-produced neutron diagnostic by radiative capture gamma-rays

Review of Scientific Instruments 89, 023505 (2018); https://doi.org/10.1063/1.5019228

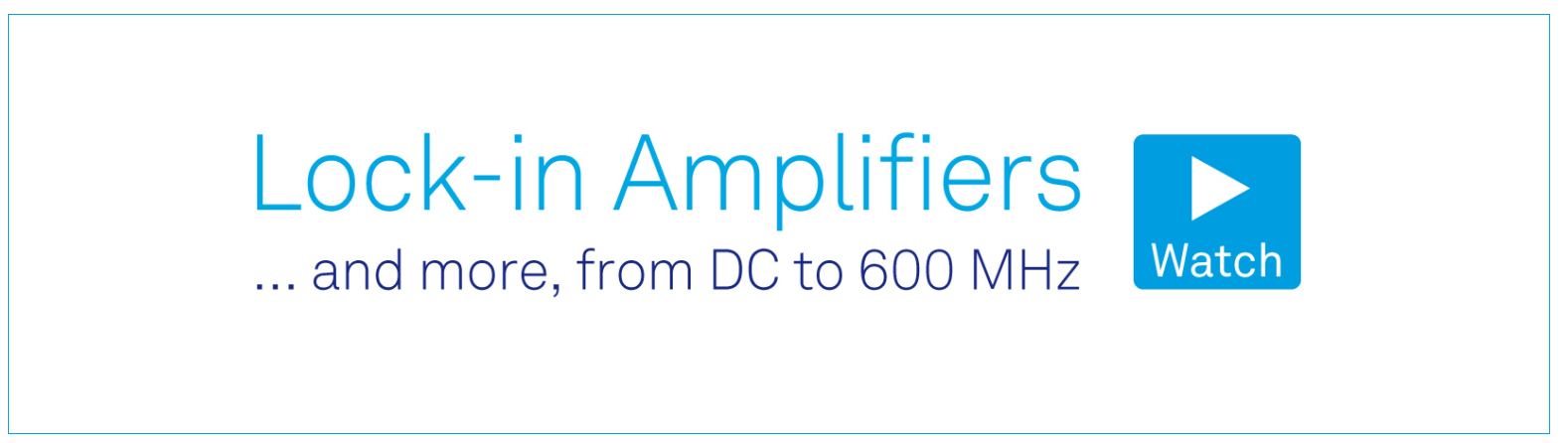

... and more, from DC to $600 \mathrm{MHz}$ Watch 


\title{
Spectral characterization of laser-accelerated protons with CR-39 nuclear track detector
}

\author{
M. Seimetz, ${ }^{1, a)}$ P. Bellido, ${ }^{1}$ P. García, ${ }^{1}$ P. Mur, ${ }^{1}$ A. Iborra, ${ }^{1}$ A. Soriano, ${ }^{1,2}$ T. Hülber, ${ }^{3}$ \\ J. García López, ${ }^{4}$ M. C. Jiménez-Ramos, ${ }^{5}$ R. Lera, ${ }^{6}$ A. Ruiz-de la Cruz, ${ }^{6}$ I. Sánchez, ${ }^{6}$ \\ R. Zaffino, ${ }^{7}$ L. Roso, ${ }^{8}$ and J. M. Benlloch ${ }^{1}$ \\ ${ }^{1}$ Instituto de Instrumentación para Imagen Molecular (I3M), CSIC-Universitat Politècnica de València, \\ Camino de Vera s/n, Ed. $8 B-N-1 a, 46022$ Valencia, Spain \\ ${ }^{2}$ Departament d'Informàtica, Universitat de València, Av. de la Universitat s/n, 46100 Burjassot, Spain \\ ${ }^{3}$ Radosys Kft., Vegyész u. 17-27, 1116 Budapest, Hungary \\ ${ }^{4}$ Departamento Física Atómica, Molecular y Nuclear, Universidad de Sevilla, Av. Reina Mercedes s/n, \\ 41012 Sevilla, Spain \\ ${ }^{5}$ Centro Nacional de Aceleradores (CNA), U. Sevilla-J. Andalucía-CSIC, Avda. Thomas Alva Edison 7, \\ 41092 Sevilla, Spain \\ ${ }^{6}$ Proton Laser Applications S.L. (PLA), Avda. Vilafranca del Penedès 11, 08734 Olèrdola, Spain \\ ${ }^{7}$ Instituto de Microelectrónica de Barcelona (IMB-CNM, CSIC), C/ dels Til.lers Campus UAB, \\ 08193 Cerdanyola del Vallès (Barcelona), Spain \\ ${ }^{8}$ Centro de Láseres Pulsados (CLPU), Calle del Adaja, 37185 Villamayor, Spain
}

(Received 17 October 2017; accepted 22 January 2018; published online 7 February 2018)

\begin{abstract}
CR-39 nuclear track material is frequently used for the detection of protons accelerated in laserplasma interactions. The measurement of track densities allows for determination of particle angular distributions, and information on the kinetic energy can be obtained by the use of passive absorbers. We present a precise method of measuring spectral distributions of laser-accelerated protons in a single etching and analysis process. We make use of a one-to-one relation between proton energy and track size and present a precise calibration based on monoenergetic particle beams. While this relation is limited to proton energies below $1 \mathrm{MeV}$, we show that the range of spectral measurements can be significantly extended by simultaneous use of absorbers of suitable thicknesses. Examples from laser-plasma interactions are presented, and quantitative results on proton energies and particle numbers are compared to those obtained from a time-of-flight detector. The spectrum end points of continuous energy distributions have been determined with both detector types and coincide within 50-100 keV. Published by AIP Publishing. https://doi.org/10.1063/1.5009587
\end{abstract}

\section{INTRODUCTION}

The acceleration of protons by highly intense pulses of femtosecond lasers is a field of very active research with many potential applications. ${ }^{1}$ Many experimental results obtained in interactions of lasers focused on thin targets, with intensities between $10^{18}$ and $10^{21} \mathrm{~W} / \mathrm{cm}^{2}$, have been interpreted in the framework of Target Normal Sheath Acceleration $(\text { TNSA) })^{2,3}$ although different acceleration mechanisms have been observed as well. ${ }^{4}$ The spectral distribution of the protons varies strongly with the experimental conditions, and its precise measurement is an essential part of related experiments. A big challenge resides in the simultaneous detection of a large number of particles (of the order $10^{8} \mathrm{sr}^{-1}$ ) which are produced on a time scale of picoseconds and whose energies are typically spread over a broad interval up to a certain maximum. For a quantitative characterization, protons of different energies may be separated spatially ${ }^{5}$ or by the time-of-flight technique. ${ }^{6}$ In addition, passive detector materials have often been used due to their easy handling and their lack of sensitivity to electromagnetic noise. ${ }^{7}$ These comprise radiochromic

\footnotetext{
a) Author to whom correspondence should be addressed: mseimetz@i3m. upv.es
}

films which allow for highly resolved analysis of the beam profile ${ }^{8,9}$ and CR-39 samples, ${ }^{10,11}$ the latter being especially useful at small particle densities and for a clear separation between protons and ions from electrons and photons.

CR-39 [polyallyl-diglycol-carbonate (PADC), $\mathrm{C}_{12} \mathrm{H}_{18} \mathrm{O}_{7}$ ] has been applied in different ways for the detection and energy measurement of laser-accelerated protons. Multi-MeV protons can be absorbed in CR-39 stacks, possibly with additional, passive materials in front, in order to obtain their energies from the range and corresponding energy loss. ${ }^{9,12,13}$ Jeong et al. ${ }^{14}$ recently described a method to analyze the pit sizes on the front and back sides of CR-39 samples to extend the proton energy range beyond $10 \mathrm{MeV}$. For protons between 0.9 and $2.5 \mathrm{MeV}$, a method based on multiple etching has been proposed recently. ${ }^{15}$ However, for practical purposes, a single processing cycle (etching and track analysis) of CR-39 samples is preferred, especially for elevated numbers of samples. The main objective of the present work is to determine particle energies directly from the track sizes. Previous studies showed that above $1 \mathrm{MeV}$ approximately the diameters of etched tracks become smaller with increasing proton energy as the ionisation density decreases. ${ }^{16}$ The opposite tendency, an increase in track size toward higher energies, has been observed below $1 \mathrm{MeV}$ in some publications, indicating a strong dependence 
on the etching conditions. Lee et al. ${ }^{17}$ measured a maximum track diameter of $14.3 \mu \mathrm{m}$ for $0.3 \mathrm{MeV}$ protons after $6 \mathrm{~h}$ etching at $70{ }^{\circ} \mathrm{C}$ in $6 \mathrm{~N} \mathrm{NaOH}$. Under the same conditions, similar observations have been made more recently by Baccou et al. ${ }^{18}$ A more abundant calibration was presented by Malinowska and co-workers ${ }^{19}$ for a $6.25 \mathrm{~N} \mathrm{NaOH}$ bath at $70{ }^{\circ} \mathrm{C}$, finding a clear relation between the maximum track size and the etching time (4-20 h).

Inspired mainly by this latter publication, we have sought for a method of extracting quantitative proton spectra directly from the distribution of track sizes on single CR-39 samples. This requires a one-to-one relation between track diameters and proton energies. This work focuses on proton energies between 0.2 and $5.5 \mathrm{MeV}$ corresponding to the expected cinematic range at a few TW laser power. In order to establish the desired relation, we have irradiated CR-39 samples with monoenergetic protons from a tandem accelerator (Sec. II). For practical use, it is necessary to guarantee a limited processing time after irradiation of the samples, including etching, scanning, and image processing; particle spectra should be extracted within a few hours after a laser-acceleration experiment. We have evaluated different etching conditions and image analysis procedures to find a suitable procedure (Sec. III). Finally, we have used this procedure to analyze samples of laser-accelerated protons (Sec. IV).

\section{SAMPLE IRRADIATION WITH MONOENERGETIC PROTON BEAMS}

All experiments of the present study have been carried out with CR-39 samples of $10 \times 10 \mathrm{~mm}^{2}$ size and $0.9 \mathrm{~mm}$ thickness. An etching bath container with remote controlled temperature has been manufactured by the provider of the detector material (Radosys, Hungary). The nuclear track detectors have been etched with $6.25 \mathrm{~N} \mathrm{NaOH}$ at $90{ }^{\circ} \mathrm{C}$ with varying etching times (2-6 h). One control series has been etched at $70{ }^{\circ} \mathrm{C}$ during $4 \mathrm{~h}$. The deviation of the mean temperature from the nominal temperature throughout the overall etching time is typically below $\pm 1^{\circ} \mathrm{C}$.

Calibration data with monoenergetic proton beams have been taken at the $3 \mathrm{MV}$ tandem accelerator of the Spanish National Accelerator Centre (CNA, Seville). The beam energy can be freely adjusted between 0.7 and $5.5 \mathrm{MeV}$, with a precision of a few $\mathrm{keV}$. In order to extend the proton energy range below $0.7 \mathrm{MeV}$, mylar foils with a total thickness of $10 \mu \mathrm{m}$ have been placed in front of the CR-39 samples. The resulting energies and their corresponding uncertainties in this interval are between $(100 \pm 49) \mathrm{keV}$ and $(508 \pm 33) \mathrm{keV}$ as calculated with the software SRIM (The Stopping and Range of Ions in Matter). ${ }^{20} \mathrm{~A}$ total of 19 different beam energies have been selected in the overall interval (0.1-5.5 MeV), and several samples have been irradiated for each one.

The particle flux has been controlled in order to avoid saturation due to a large number of overlapping tracks. The minimum measurable beam current of the tandem accelerator in continuous operation is about $1 \mathrm{pA}$ or $6 \times 10^{6}$ protons per second which are distributed over an area of $1 \mathrm{~cm}^{2}$ defined by a rhombic collimator. The beam profile was not uniform. A beam kicker between the proton source and the tandem, coupled to a pulse generator, has been used to produce 0.1-10 ms long bunches at $1 \mathrm{~Hz}$, reducing the mean particle number by a factor $10^{2}-10^{4}$. A beam monitor based on a plastic scintillator with photomultiplier tube (PMT) readout on a fast oscilloscope ${ }^{21}$ allowed for direct verification of the proton flux in the target spot. This control measurement was carried out after beam optimisation at each energy setting, before moving the samples into the beam position. Thus, every CR-39 sample was hit by a total of $10^{3}-10^{4}$ protons within a few seconds of irradiation time, resulting in sufficient numbers of non-overlapping tracks to allow for statistically significant analysis of typical diameters corresponding to each beam energy.

\section{SCANNING PROCEDURES AND IMAGE PROCESSING}

The measurement of the track size and particle numbers requires scanning of major parts of the irradiated surfaces and results in elevated numbers of images for each single CR-39 sample. This has been achieved with a nuclear track reader (Radosys PT10). The pixel size of $0.6 \mu \mathrm{m}$ is far below the diameter of typical proton tracks. We have developed a software code based on ROOT $^{22}$ for the identification and size measurement of tracks on these images over a wide range of diameters. It comprises an edge detection algorithm for the search of track borders. A Hough transform is used to identify circular patterns (track ellipticity is not considered as particles impinge at normal angle in all our experiments). It is iterated over a predefined range of diameters to search for the best match.

Representative subsets of images have been processed for each proton energy (examples are shown in Fig. 1), and the corresponding distributions of measured track diameters are represented in Fig. 2. They typically show clear peaks around a central value. For each setting, the mean value and standard deviation around the peak are interpreted as the nominal track size and its corresponding experimental error, respectively. Several factors contribute to the finite width of these peaks, the most important one being true differences in the track sizes even of "monoenergetic" samples. Between 0.2 and $0.5 \mathrm{MeV}$, these variations mainly stem from the inherent differences of proton energies due to straggling in the absorber foils. Some particles may be scattered on the collimator edges and lose part of their energy. No clear tracks of equal size have been identified at $0.1 \mathrm{keV}$.

Mean track diameters, $D_{t}$, increase rapidly with proton energy, $E_{p}$, for energies in the $0.2-1 \mathrm{MeV}$ range (referred to as "interval $I_{1}$ "). A maximum diameter is observed around $1 \mathrm{MeV}$; its position slightly varies according to the etching conditions. Beyond this maximum and up to $5.5 \mathrm{MeV}$ (interval $I_{2}$ ), protons of higher energies produce smaller tracks [Fig. 3(a)]. The turning point may be understood due to the changeover between the conical etch phase (with the pit depth below the proton range inside the CR-39 material) and the spherical phase where the pit depth exceeds the proton range. These observations are qualitatively consistent with previous publications, especially by Malinowska et al. ${ }^{19}$ (the calibrated range by Sinenian and co-workers ${ }^{16}$ started from $1 \mathrm{MeV}$, 


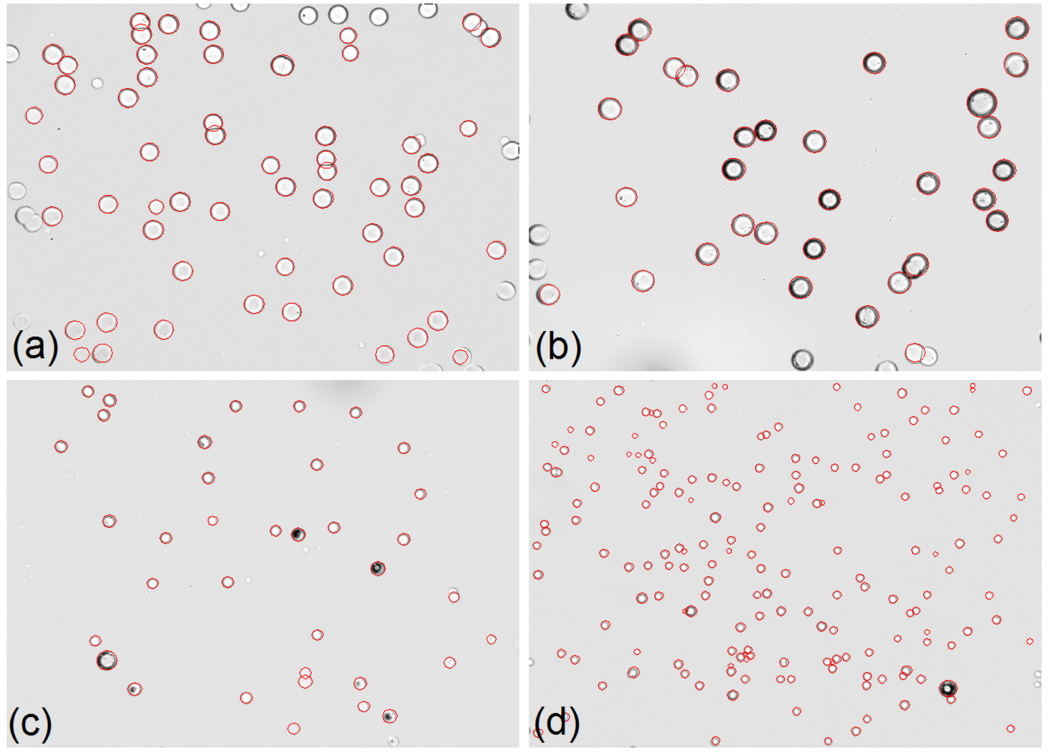

FIG. 1. Proton tracks of calibration samples for (a) $0.7 \mathrm{MeV}$, (b) $1.0 \mathrm{MeV}$, (c) $2.35 \mathrm{MeV}$, and (d) $4.5 \mathrm{MeV}$ beam energy, respectively, etched at $90{ }^{\circ} \mathrm{C}$ during $4 \mathrm{~h}$. comprising only the decreasing interval). The principal, quantitative difference resides in the large pit sizes due to the high etching temperature. At $90^{\circ} \mathrm{C}$, track diameters up to $26 \mu \mathrm{m}$ are reached after $4 \mathrm{~h}$; at $70{ }^{\circ} \mathrm{C}$, about $20 \mathrm{~h}$ are necessary to obtain similar values. ${ }^{19}$ This implies a strong gradient, $d E_{p} / d D_{t}$ $\simeq 2.5 \mu \mathrm{m} /(100 \mathrm{keV})$, in $I_{1}$ allowing for a clear separation of different proton energies with $0.6 \mu \mathrm{m}$ wide image pixels. The gradient is much smaller after $2 \mathrm{~h}$ etching, with a maximum of $16 \mu \mathrm{m}$ diameter. In turn, after $6 \mathrm{~h}$, the track identification does not further improve, and the number of overlapping tracks increases [Fig. 3(b)]. We therefore have selected the $4 \mathrm{~h}$ sample as our reference for the remainder of this study.

The data trends of the two intervals have been approximated by simple analytical functions,

$$
D_{t}=a_{0}+a_{1} E_{p}+a_{2} E_{p}^{2}
$$

for $I_{1}\left(E_{p}=0.2-1 \mathrm{MeV}\right)$ and

$$
D_{t}=b_{0}+b_{1} e^{-b_{2} E_{p}}
$$

for $I_{2}\left(E_{p}=1-5.5 \mathrm{MeV}\right)$. The data point at $1.0 \mathrm{MeV}$ forms part of both fitted samples. The results for the $4 \mathrm{~h}$ data set are

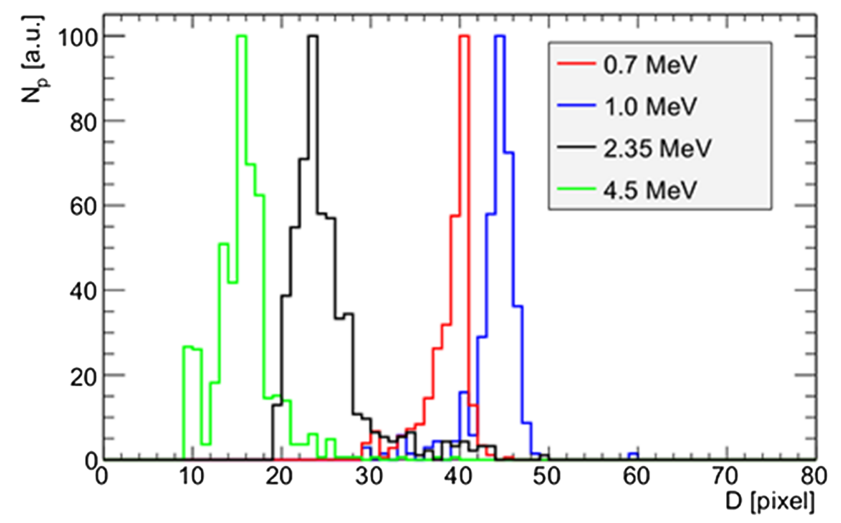

FIG. 2. Track diameter distributions of calibration samples. For illustration, the counts have been normalised to the same maximum height. shown in Fig. 3 together with the 95\% confidence intervals; the fit parameters are summarized in Table I. Equation (1) can be inverted to obtain proton energies from the measured track diameters,

$$
E_{p}\left(D_{t}\right)=\sqrt{\frac{D_{t}-a_{0}}{a_{2}}+\left(\frac{a_{1}}{2 a_{2}}\right)^{2}}-\frac{a_{1}}{2 a_{2}} .
$$
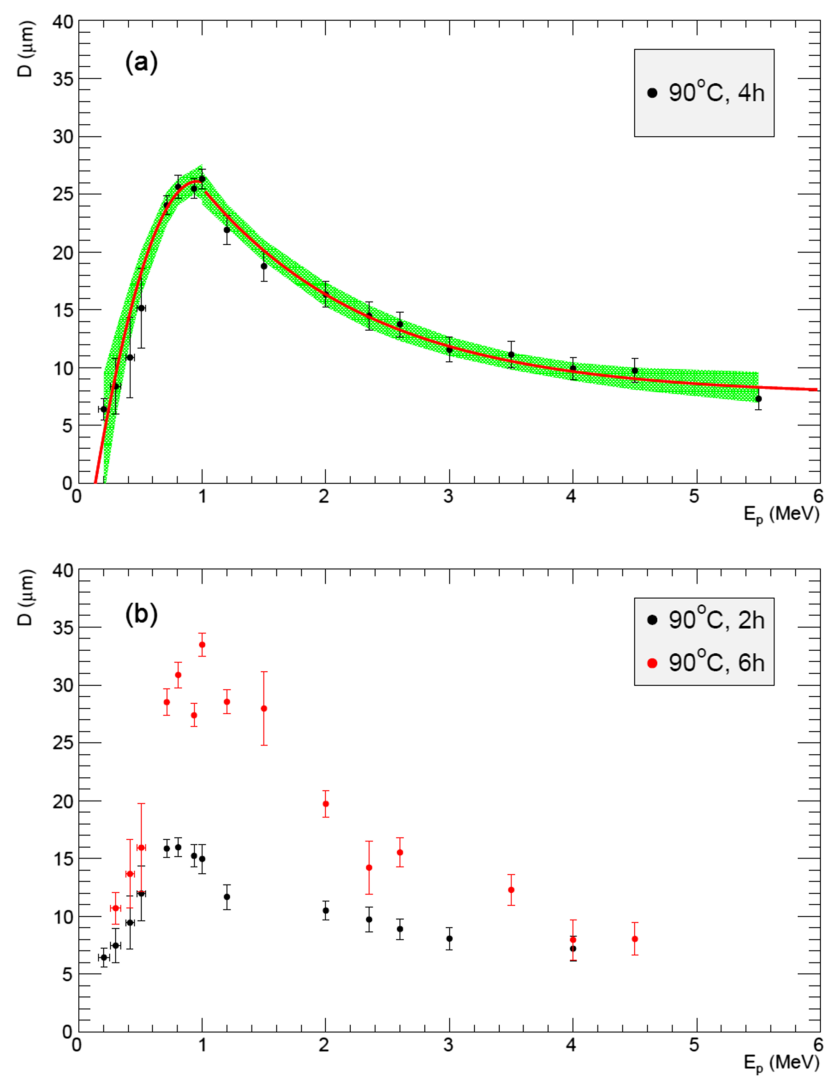

FIG. 3. Measured track diameters as a function of proton energy. (a) $4 \mathrm{~h}$ etching at $90{ }^{\circ} \mathrm{C}$. (b) $2 \mathrm{~h}$ and $6 \mathrm{~h}$ at $90^{\circ} \mathrm{C}$. 
TABLE I. Fitted parameters for the sample of Fig. 3(a).

\begin{tabular}{llll}
\hline \hline$a_{0}$ & $-9.24 \pm 4.61$ & $b_{0}$ & $7.61 \pm 1.12$ \\
$a_{1}$ & $73.63 \pm 13.69$ & $b_{1}$ & $36.92 \pm 4.19$ \\
$a_{2}$ & $-38.35 \pm 9.53$ & $b_{2}$ & $0.722 \pm 0.134$ \\
\hline
\end{tabular}

Obviously, this holds only as long as all protons incident on the CR-39 samples have kinetic energies below $1 \mathrm{MeV}$. Despite this limitation, the proposed technique can provide precise particle spectra as will be shown in Sec. IV.

\section{APPLICATION TO LASER-ACCELERATED PROTONS}

We have used the CR-39 samples of $1 \mathrm{~cm}^{2}$ size for the spectral characterization of protons from laser-plasma interactions. These experiments have been carried out on a $3 \mathrm{TW}$, table-top Ti:sapphire laser with up to $165 \mathrm{~mJ}$ focused energy on the laser target in 55 fs pulses (details of the setup have been reported in Ref. 23). To illustrate our method, we present three experimental data sets with different, maximum proton energies, obtained due to differing laser energies and target compositions. Consider first a single laser shot at low intensity (93 mJ on a $7 \mu \mathrm{m}$ thick aluminum foil, "example 1 "). Figure 4(a) shows an image of a CR-39 sample placed $230 \mathrm{~cm}$ behind the target and directly exposed to the particle flux from a single laser shot. The etching conditions and image processing are as described in Sec. III, resulting in a distribution of track diameters represented in Fig. 4(b). For the smallest track sizes, relatively large differences between neighbouring bins can be seen. This results from finite binning effects of the automatic track recognition algorithm. The major part of the distribution is smooth. The track diameters are converted into proton energies, Eq. (3), as shown in Fig. 5. The binning of this spectral distribution corresponds to the pixel size of the initial image. As the conversion to the energy scale is nonlinear, the final bins are not of equal width as is indicated by the horizontal error bars. The vertical error bars correspond to the square root of counts of each bin in Fig. 4(b). A scale factor taking into account the total area of analyzed, microscopic images and the target distance is applied to obtain absolute particle numbers per solid angle. The spectral shape can be approximated by an exponential decrease up to a sharp maximum energy, $E_{\max }$, which is typical of protons accelerated in laser-plasma

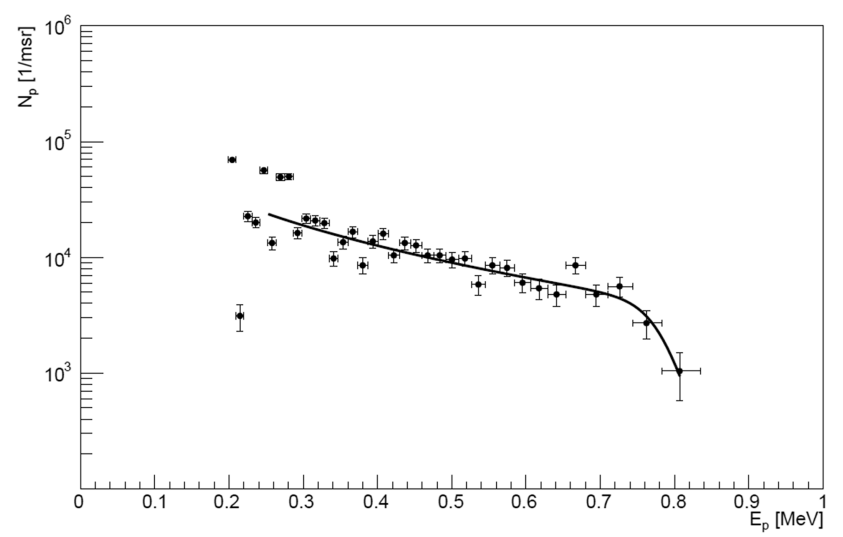

FIG. 5. Spectral distribution of protons extracted from the CR-39 sample of Fig. 4.

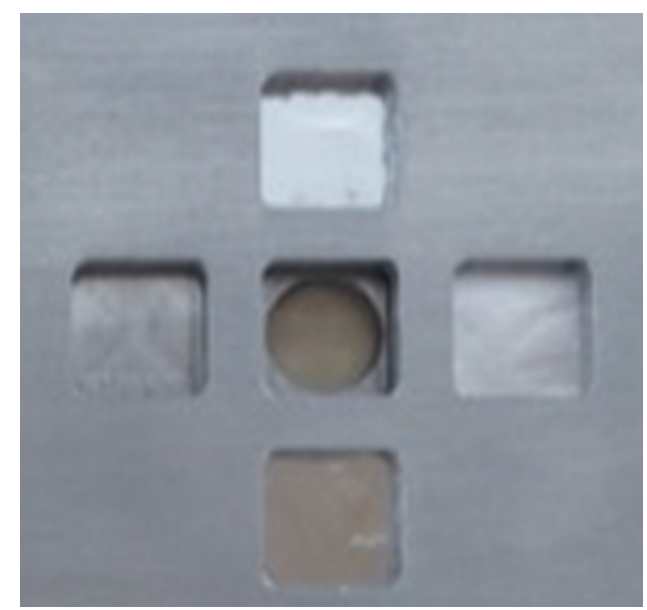

FIG. 6. Support structure for four individual CR-39 samples, each one covered with aluminum foils of different thicknesses. Part of the protons pass through the central hole and hit the TOF detector behind.

interactions like TNSA. The number of protons, $N_{p}$, of energy $E_{p}$ may be represented analytically by

$$
N_{p}=\frac{N_{0}}{E_{p}} \cdot e^{-E_{p} / E_{0}} /\left(1+e^{\left(E_{p}-E_{\max }\right) / \Delta E}\right) .
$$

Here, $E_{0}$ is interpreted as the mean temperature of a Boltzmann-like distribution. ${ }^{8}$ The parameter $\Delta E$ has been introduced in the term in parentheses to reproduce the finite (a)

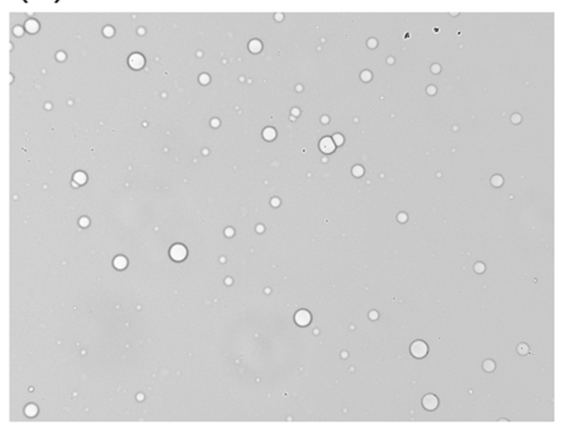

(b)

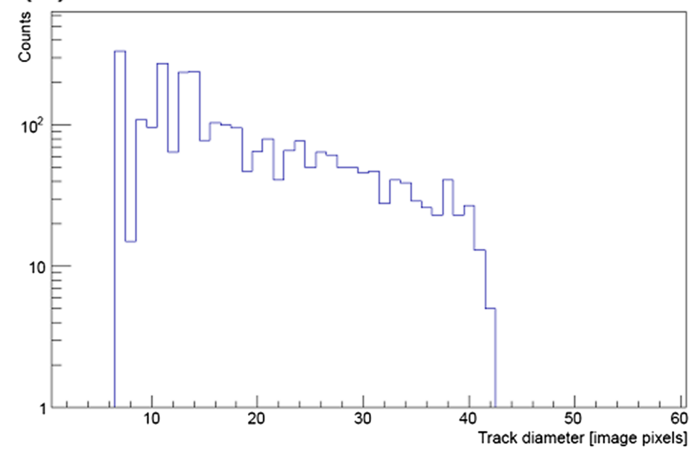

FIG. 4. (a) Microscopic image of a CR-39 sample without absorber foil, exposed to a single laser shot at low intensity (example 1). (b) Distribution of track diameters obtained from a series of images of the same sample. 
TABLE II. Minimum and maximum incident proton energies on aluminum foils of thickness $d_{\mathrm{abs}}$ corresponding to the calibrated range of detected energies on CR-39 samples.

\begin{tabular}{lcc}
\hline \hline$d_{\mathrm{abs}} / \mu \mathrm{m}$ & $E_{\min } / \mathrm{keV}$ & $E_{\mathrm{max}} / \mathrm{keV}$ \\
\hline 4 & 525 & 1200 \\
7 & 750 & 1300 \\
10 & 875 & 1400 \\
12.5 & 990 & 1500 \\
18 & 1225 & 1700 \\
25 & 1500 & 1925 \\
35 & 1850 & 2200 \\
43 & 2100 & 2400 \\
50 & 2275 & 2600 \\
\hline \hline
\end{tabular}

width of the fall-off. In this case, $E_{0}=(868.4 \pm 163.4) \mathrm{keV}$, $E_{\max }=(781.8 \pm 12.3) \mathrm{keV}$, and $\Delta E=(22.0 \pm 8.7) \mathrm{keV}$ have been obtained from a fit to the data.

At low laser intensity like the measurement described earlier, the proton energies were found to be well within the turning point of the calibration curve (below $1 \mathrm{MeV}$ ). For tests at higher laser energy (165 mJ on target), our setup has been slightly modified. For each laser shot, four CR-39 samples have been arranged around a central hole (Fig. 6) to allow for simultaneous observation of protons from the same shot on a time-of-flight (TOF) detector. ${ }^{21}$ The opening angle of the proton beam (typically around $10^{\circ}$ ) is much larger than the lateral displacement of the samples $\left(\sim 1^{\circ}\right)$; thus, we expect to observe protons of very similar spectral distributions on the CR-39 and TOF detectors. The target distance was $100 \mathrm{~cm}$. In addition, the CR-39 samples have been covered with aluminum foils of different thicknesses, for two purposes. On the one hand, to eliminate the dominant, low-energetic part of the continuous spectral distributions and thus avoid saturation due to overlapping tracks. And on the other hand, to reduce the energy of the remaining particles on the detector surface, $E_{p}^{\mathrm{det}}$, to the calibrated range below $1 \mathrm{MeV}$. The corresponding energy loss inside the foils, $E_{\mathrm{abs}}$, has been calculated with SIMNRA. ${ }^{24}$ For a given absorber thickness, $d_{\mathrm{abs}}$, incident protons must possess a minimum kinetic energy, $E_{\min }$, to exceed the detection limit on the subsequent CR-39 sample (200 keV) and produce clear tracks. Similarly, the initial energy of the protons, $E_{p}$, must not exceed a maximum, $E_{\max }$, to make sure that they fall inside the calibrated range below $1 \mathrm{MeV}$ (Table II). For each measured track, the proton energy on the detector sample is calculated

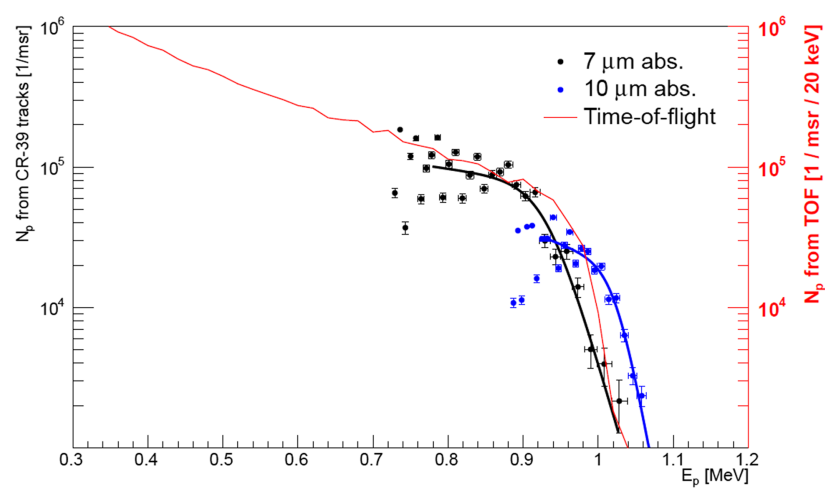

FIG. 8. Comparison of proton spectra extracted from CR-39 samples covered with $7 \mu \mathrm{m}$ and $10 \mu \mathrm{m}$ aluminum foils, respectively. The thin curve shows the spectra of the same shot reconstructed from the TOF detector.

from its diameter, Eq. (3), and the initial energy is obtained from

$$
E_{p}=E_{p}^{\mathrm{det}}\left(D_{t}\right)+E_{\mathrm{abs}}\left(d_{\mathrm{abs}}, E_{p}^{\mathrm{det}}\right) .
$$

As an example for this method, consider CR-39 samples obtained from a single laser shot on a $2 \mu \mathrm{m}$ thick mylar target foil ("example 2"). Individual tracks can clearly be distinguished when $7 \mu \mathrm{m}$ or $10 \mu \mathrm{m}$ thick absorbers are used (Fig. 7). With aluminum foils of $4 \mu \mathrm{m}$ thickness or less, to the contrary, the track density is too large for a precise measurement of their diameters. The minimum absorber thickness necessary to avoid saturation depends on the exact conditions of each laser shot. Therefore we have used four different samples at the same time in order to obtain one or two at least with non-overlapping tracks over a wide range of diameters which allow for spectral reconstruction close to the maximum proton energy. Following the procedure described in Sec. III, the distribution of track diameters is obtained for a representative selection of microscopic images of each sample. The pit diameters are converted into proton energies incident on the CR-39 samples using Eq. (3). Subsequently, the initial particle energy (from the laser-plasma interaction) is calculated correcting for the energy loss in the absorber.

In Fig. 8, the data points from a CR-39 sample covered with a $7 \mu \mathrm{m}$ thick absorber start around $750 \mathrm{keV}$ as stated in Table II. They correspond to proton numbers of the order $10^{5} \mathrm{msr}^{-1}$ up to $0.93 \mathrm{MeV}$ where a sudden decrease is observed. The same analysis has been performed for the sample covered with a $10 \mu \mathrm{m}$ thick absorber. Here, the overall (a)

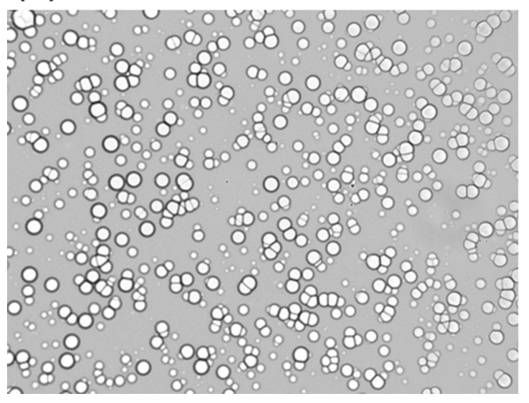

(b)

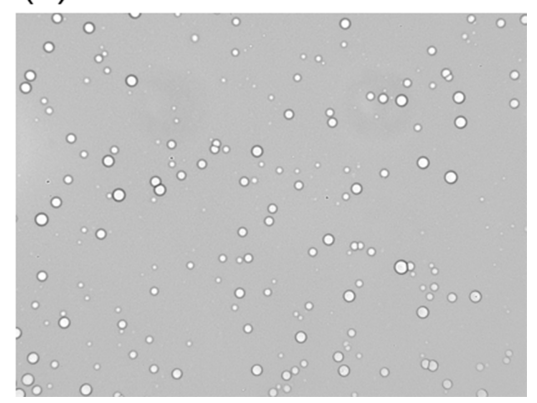

FIG. 7. Microscopic images of CR-39 samples after a single laser shot (example 2), covered with (a) $7 \mu \mathrm{m}$ and (b) $10 \mu \mathrm{m}$ of aluminum, respectively. 
(a)

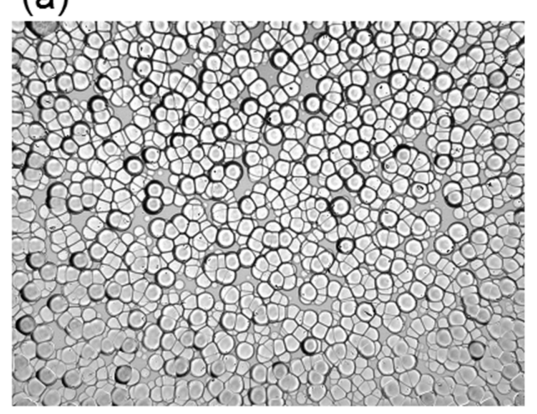

(b)

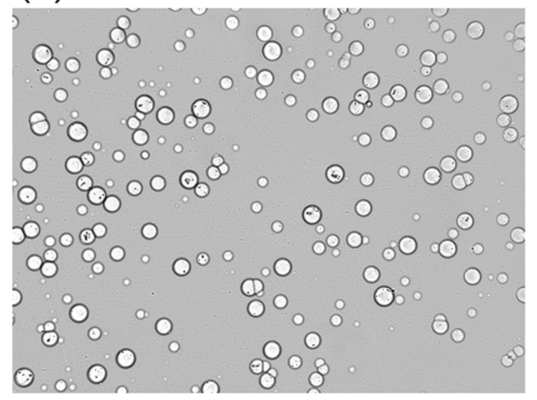

FIG. 9. Microscopic images of CR-39 samples exposed to a single laser shot (example 3), covered with (a) $25 \mu \mathrm{m}$ and (b) $35 \mu \mathrm{m}$ of aluminum, respectively. range of detected particle energies is smaller (starting from $875 \mathrm{keV}$ ) and the particle numbers per bin decrease rapidly at $1.02 \mathrm{MeV}$. Both spectra are compared to the particle energy distribution which has been obtained for the same laser shot using the time-of-flight detector (continuous line in Fig. 7; for details of the analysis and normalization procedure, see Ref. 23). The three independent measurements give similar values for the maximum proton energy within a full range of $(0.98 \pm 0.05) \mathrm{MeV}$.

In the previous example, the highest particle energies were still close to the range allowing for a one-to-one correspondence between track diameters. We have studied the applicability of our method also for the case of significantly higher proton energies. This is demonstrated with a single laser shot of $165 \mathrm{~mJ}$ on a $0.65 \mu \mathrm{m}$ thin aluminum membrane produced with MEMS techniques from a silicon wafer ("example 3"). Four CR-39 samples have been arranged as described earlier but covered with thicker absorber foils (18-43 $\mu \mathrm{m}$ of aluminum). With $18 \mu \mathrm{m}$ and $25 \mu \mathrm{m}$ covers, the track density is too high for a spectral analysis, Fig. 9(a). With a $35 \mu \mathrm{m}$ absorber, to the contrary, individual tracks with a wide range of diameters can be observed [Fig. 9(b)]. The spectra of Fig. 10 have been reconstructed following the previously described procedure. Here again, the maximum proton energy $(2.08 \mathrm{MeV})$ compares well with the result of the time-of-flight measurement of the same laser shot. In this case, only the region close to the end point of the spectral distribution can be investigated by our method which, however, allows for an

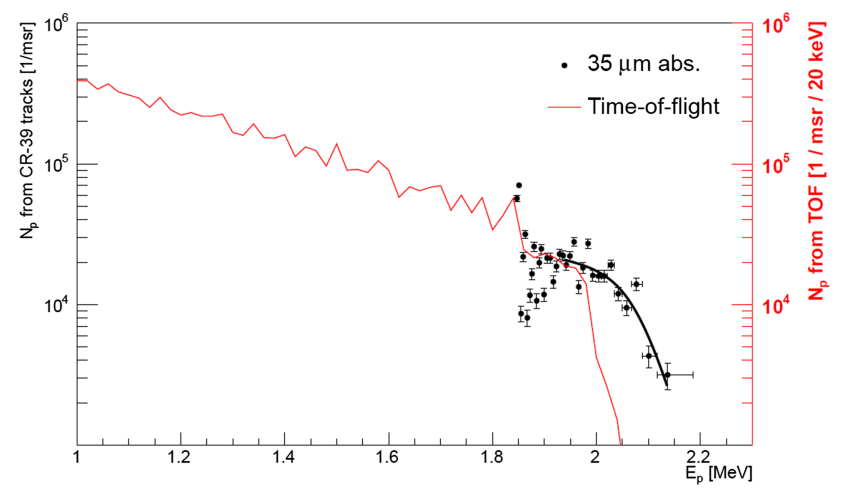

FIG. 10. Proton spectra extracted from CR-39 samples covered with a $35 \mu \mathrm{m}$ aluminum foil (example 3). The thin line shows the spectra of the same shot reconstructed from the TOF detector. independent check of the results obtained with a different type of detector.

In laser acceleration based on TNSA, generally more than one ion species are observed. Among these, protons carry the highest kinetic energies (in $\mathrm{MeV} / \mathrm{u}$ ) due to their high charge/mass ratio. ${ }^{25}$ In the data of examples 2 and 3 above, recorded by the TOF detector, we have found indications of ions heavier than protons, however far from the leading signal edge of flight times. They can be interpreted as carbon ions with maximum energies of 4-4.5 MeV (less than $0.4 \mathrm{MeV} / \mathrm{u}$ ). The range of such ions in aluminum absorbers is about $4 \mu \mathrm{m}$ to the most. Therefore we can rule out that particles other than protons may reach our CR-39 samples and produce tracks that might be falsely interpreted. Nevertheless, for the applicability of our method of obtaining proton spectra, it is always important to check the possible admixture of heavy ions, e.g., by measuring the deflection in magnetic fields. ${ }^{11}$

\section{v. CONCLUSIONS}

We present a method of determining spectral properties of laser-accelerated protons using the CR-39 track detector. Proton energies between $0.2 \mathrm{MeV}$ and $1.0 \mathrm{MeV}$ are directly correlated with the track diameters. Etching at $90{ }^{\circ} \mathrm{C}$ during $4 \mathrm{~h}$ allows for fully exploiting this equivalence as we have shown in calibration measurements. We have applied our method to laser-accelerated protons in different working regimes. At low laser intensities where particle energies do not exceed $1 \mathrm{MeV}$, precise spectra can be extracted from one irradiated CR-39 sample and after a single etching and image analysis procedure. At higher intensities and correspondingly elevated particle energies and track densities, several CR-39 samples can be covered with absorber foils of different thicknesses to shift the detected part of the spectra to the calibrated range. This allows for a precise determination of the maximum proton energies and the spectral distribution of the most energetic particles. The information obtained in this way is much more detailed than "binary" measurements based on the mere observation of tracks behind absorbers of different thicknesses. An extension of our analysis method toward higher track densities, as suggested by Gaillard et al. ${ }^{26}$ may allow for the characterization of larger energy intervals.

The proposed method may be limited to maximum proton energies of a few $\mathrm{MeV}$ and, correspondingly, moderate laser energies $(\leq 1 \mathrm{~J})$ as straggling in the absorbers becomes 
increasingly important at larger thicknesses. Nevertheless, it can be a useful tool for experiments in a regime accessible to cost-effective, table-top lasers. An interesting application consists of the simultaneous measurement of spectral and angular distributions (in other words, the variation of spectra as a function of the detection angle) by covering a larger area with CR-39 samples. Further, it allows for cross-checks of results obtained from alternative detection techniques, a task which is still challenging at high particle intensities in very short pulses.

\section{ACKNOWLEDGMENTS}

This work has been developed in close collaboration with Radosys Ltd. (Budapest, Hungary) which provided CR-39 detector material, etching bath container, and readout equipment. The authors highly appreciate the fruitful contribution of the CNA accelerator operators. This project has been funded by the Spanish Ministry for Economy and Competitiveness within the Retos-Colaboración 2015 initiative, Ref. No. RTC2015-3278-1. P. Mur has been awarded a Garantía Juvenil grant.

${ }^{1}$ H. Daido, M. Nishiuchi, and A. S. Pirozhkov, "Review of laser-driven ion sources and their applications," Rep. Prog. Phys. 75, 056401 (2012).

${ }^{2}$ M. Roth and M. Schollmeier, "Ion acceleration-target normal sheath acceleration," CERN Yellow Rep. 1, 231 (2016).

${ }^{3}$ J. Fuchs, P. Antici, E. d'Humières, E. Lefebvre, M. Borghesi, E. Brambrink, C. Cecchetti, M. Kaluza, V. Malka, M. Manclossi, S. Meyroneinc, P. Mora, J. Schreiber, T. Toncian, H. Pépin, and P. Audebert, "Laser-driven proton scaling laws and new paths towards energy increase," Nat. Phys. 2, 48-54 (2006).

${ }^{4}$ J. Schreiber, P. R. Bolton, and K. Parodi, "Invited review article: 'Hands-on' laser-driven ion acceleration: A primer for laser-driven source development and potential applications," Rev. Sci. Instrum. 87, 071101 (2016).

${ }^{5}$ R. Prasad, D. Doria, S. Ter-Avetisyan, P. Foster, K. Quinn, L. Romagnani, C. Brenner, J. Green, P. Gallegos, M. Streeter, D. Carroll, O. Tresca, N. Dover, C. Palmer, J. Schreiber, D. Neely, Z. Najmudin, P. McKenna, M. Zepf, and M. Borghesi, "Calibration of Thomson parabola-MCP assembly for multi-MeV ion spectroscopy," Nucl. Instrum. Methods Phys. Res., Sect. A 623, 712-715 (2010).

${ }^{6}$ A. Yogo, H. Daido, A. Fukumi, Z. Li, K. Ogura, A. Sagisaka, A. S. Pirozhkov, S. Nakamura, Y. Iwashita, T. Shirai, A. Noda, Y. Oishi, T. Nayuki, T. Fujii, K. Nemoto, I. W. Choi, J. H. Sung, D.-K. Ko, J. Lee, M. Kaneda, and A. Itoh, "Laser prepulse dependency of proton-energy distributions in ultraintense laser-foil interactions with an online time-of-flight technique," Phys. Plasmas 14, 043104 (2007).

${ }^{7}$ P. Bolton, M. Borghesi, C. Brenner, D. Carroll, C. D. Martinis, A. Flacco, V. Floquet, J. Fuchs, P. Gallegos, D. Giove, J. Green, S. Green, B. Jones, D. Kirby, P. McKenna, D. Neely, F. Nuesslin, R. Prasad, S. Reinhardt, M. Roth, U. Schramm, G. Scott, S. Ter-Avetisyan, M. Tolley, G. Turchetti, and J. Wilkens, "Instrumentation for diagnostics and control of laseraccelerated proton (ion) beams," Phys. Med. 30, 255-270 (2014).

${ }^{8}$ F. Nürnberg, M. Schollmeier, E. Brambrink, A. Blažević, D. C. Carroll, K. Flippo, D. C. Gautier, M. Geißel, K. Harres, B. M. Hegelich, O. Lundh, K. Markey, P. McKenna, D. Neely, J. Schreiber, and M. Roth, "Radiochromic film imaging spectroscopy of laser-accelerated proton beams," Rev. Sci. Instrum. 80, 033301 (2009).

${ }^{9}$ C. Scullion, D. Doria, L. Romagnani, H. Ahmed, A. Alejo, O. Ettlinger, R. Gray, J. Green, G. Hicks, D. Jung, K. Naughton, H. Padda, K. Poder, G. Scott, D. Symes, S. Kar, P. McKenna, Z. Najmudin, D. Neely, M. Zepf, and M. Borghesi, "Angularly resolved characterization of ion beams from laser-ultrathin foil interactions," J. Instrum. 11, C09020 (2016).
${ }^{10}$ Y. Paudel, J. Frenje, A. Merwin, and N. R.-L. Galloudec, "CR39 imaging technique for quick track analysis of particles generated in high-intensity laser target interactions," J. Instrum. 6, T08004 (2011).

${ }^{11}$ M. Kanasaki, S. Jinno, H. Sakaki, K. Kondo, K. Oda, T. Yamauchi, and Y. Fukuda, "The precise energy spectra measurement of laser-accelerated $\mathrm{MeV} / \mathrm{n}$-class high-Z ions and protons using CR-39 detectors," Plasma Phys. Controlled Fusion 58, 034013 (2016).

${ }^{12}$ M. Kanasaki, A. Hattori, H. Sakaki, Y. Fukuda, A. Yogo, S. Jinno, M. Nishiuchi, K. Ogura, K. Kondo, K. Oda, and T. Yamauchi, "A high energy component of the intense laser-accelerated proton beams detected by stacked CR-39," Rad. Meas. 50, 46-49 (2013).

${ }^{13}$ A. Zigler, S. Eisenman, M. Botton, E. Nahum, E. Schleifer, A. Baspaly, I. Pomerantz, F. Abicht, J. Branzel, G. Priebe, S. Steinke, A. Andreev, M. Schnuerer, W. Sandner, D. Gordon, P. Sprangle, and K. W. D. Ledingham, "Enhanced proton acceleration by an ultrashort laser interaction with structured dynamic plasma targets," Phys. Rev. Lett. 110, 215004 (2013).

${ }^{14}$ T. W. Jeong, P. K. Singh, C. Scullion, H. Ahmed, P. Hadjisolomou, C. Jeon, H. Yun, K. F. Kakolee, M. Borghesi, and S. Ter-Avetisyan, "CR-39 track detector for multi-MeV ion spectroscopy," Sci. Rep. 7, 2152 (2017).

${ }^{15}$ F. Bahrami, F. Mianji, R. Faghihi, M. Taheri, and A. Ansarinejad, "Response of CR-39 to $0.9-2.5 \mathrm{MeV}$ protons for $\mathrm{KOH}$ and $\mathrm{NaOH}$ etching solutions," Nucl. Instrum. Methods Phys. Res., Sect. A 813, 96-101 (2016).

${ }^{16}$ N. Sinenian, M. J. Rosenberg, M. Manuel, S. C. McDuffee, D. T. Casey, A. B. Zylstra, H. G. Rinderknecht, M. G. Johnson, F. H. Séguin, J. A. Frenje, C. K. Li, and R. D. Petrasso, "The response of CR-39 nuclear track detector to 1-9 MeV protons," Rev. Sci. Instrum. 82, 103303 (2011).

${ }^{17}$ J. Lee, J. Jo, S. Park, K. Lee, Y. Lee, K.-H. Yea, Y. Cha, and Y. Jeong, "Study on the tracks in a nuclear track detector (CR39) for detection of laser-induced charged particles," J. Korean Phys. Soc. 51, 426-430 (2007).

${ }^{18}$ C. Baccou, V. Yahia, S. Depierreux, C. Neuville, C. Goyon, F. Consoli, R. D. Angelis, J. E. Ducret, G. Boutoux, J. Rafelski, and C. Labaune, "CR-39 track detector calibration for $\mathrm{H}, \mathrm{He}$, and $\mathrm{C}$ ions from $0.1-0.5 \mathrm{MeV}$ up to $5 \mathrm{MeV}$ for laser-induced nuclear fusion product identification," Rev. Sci. Instrum. 86, 083307-83310 (2015).

${ }^{19}$ A. Malinowska, A. Szydłowski, M. Jaskóła, A. Korman, B. Sartowska, T. Kuehn, and M. Kuk, "Investigations of protons passing through the CR-39/PM-355 type of solid state nuclear track detectors," Rev. Sci. Instrum. 84, 073511 (2013).

${ }^{20}$ J. Ziegler and J. Biersack, SRIM-The Stopping Range of Ions in Solids (Pergamon, 1985)

${ }^{21}$ M. Seimetz, P. Bellido, A. Soriano, J. G. López, M. C. Jiménez-Ramos, B. Fernández, P. Conde, E. Crespo, A. J. González, L. Hernández, A. Iborra, L. Moliner, J. P. Rigla, M. J. Rodríguez-Álvarez, F. Sánchez, S. Sánchez, L. F. Vidal, and J. M. Benlloch, "Calibration and performance tests of detectors for laser-accelerated protons," IEEE Trans. Nucl. Sci. 62, 3216-3224 (2015).

${ }^{22}$ R. Brun and F. Rademakers, "ROOT-An object oriented data analysis framework," Nucl. Instrum. Methods Phys. Res., Sect. A 389, 81-86 (1997).

${ }^{23}$ P. Bellido, R. Lera, M. Seimetz, A. R. de la Cruz, S. Torres-Peirò, M. Galán, P. Mur, I. Sánchez, R. Zaffino, L. Vidal, A. Soriano, S. Sánchez, F. Sánchez, M. Rodríguez-Álvarez, J. Rigla, L. Moliner, A. Iborra, L. Hernández, D. Grau-Ruiz, A. González, J. García-Garrigos, E. Díaz-Caballero, P. Conde, A. Aguilar, L. Roso, and J. Benlloch, "Characterization of protons accelerated from a 3 TW table-top laser system," J. Instrum. 12, T05001 (2017).

${ }^{24}$ M. Mayer, "SIMNRA, a simulation program for the analysis of NRA, RBS and ERDA," AIP Conf. Proc. 475, 541 (1999).

${ }^{25}$ D. C. Carroll, O. Tresca, R. Prasad, L. Romagnani, P. S. Foster, P. Gallegos, S. Ter-Avetisyan, J. S. Green, M. J. V. Streeter, N. Dover, C. A. J. Palmer, C. M. Brenner, F. H. Cameron, K. E. Quinn, J. Schreiber, A. P. L. Robinson, T. Baeva, M. N. Quinn, X. H. Yuan, Z. Najmudin, M. Zepf, D. Neely, M. Borghesi, and P. McKenna, "Carbon ion acceleration from thin foil targets irradiated by ultrahigh-contrast, ultraintense laser pulses," New J. Phys. 12, 045020 (2010).

${ }^{26}$ S. Gaillard, J. Fuchs, N. R.-L. Galloudec, and T. E. Cowan, "Study of saturation of CR39 nuclear track detectors at high ion fluence and of associated artifact patterns," Rev. Sci. Instrum. 78, 013304 (2007). 